\title{
LA “GUBERNAMENTALIDAD” SISTÉMICA EN EL DOCENTE UNIVERSITARIO CONTEMPORÁNEO
}

\author{
Javier Ricardo Salcedo Casallas \\ Magister en Relaciones Internacionales \\ Docente investigador del Grupo de investigación Pedagogía, \\ Cultura y Formación Docente del Centro de Investigación en Educación y Pedagogía (CIEP) \\ Universidad de La Salle, Colombia \\ jsalcedocasallas@gmail.com \\ Cómo citar este artículo
}

Salcedo, J. R. (2013) La "Gubernamentalidad" sistémica en el docente universitario contemporáneo. Espiral, Revista de Docencia e Investigación. 3(2)

\begin{abstract}
Resumen
La noción de gubernamentalidad que aquí se expone, parte de una aprehensión metodológica foucultiana elaborada por el maestro Carlos Noguera; sin embargo, y como aporte de la investigación doctoral en reformas a la Educación Superior y Docencia universitaria que el autor de este texto está desarrollando, integra la perspectiva teórica de Niklas Luhmann o teoría de sistemas para exponer cómo en las formas nuevas de gobernar a la educación superior, el docente universitario contemporáneo se ha convertido en sistemas psíquicos de operación u operaciones de investigación y docencia, que permiten la auto referencia y el gobierno de sí y ser gobernados por un sistema de producción cognitiva desde el trabajo inmaterial de los dos sistemas psíquicos de operación docente en el marco de dos sistemas: el económico: capital humano y capital cognitivo y el de la educación superior reformada.
\end{abstract}

Palabras clave: gobernamentalidad, sistema, educación superior, sistema psíquico de percepción, docente universitario.

\footnotetext{
Abstract

The notion of governmentality is presented here is part of a methodological foucultiana apprehension made by master Carlos Noguera, however, and as a contribution of the doctoral research reforms to higher education and university teaching that the author of this text is to develop, integrate the theoretical perspective of Niklas Luhmann or systems theory to explain how new forms of governing higher education, the contemporary university teaching has become psychic systems of operation or operations research and teaching, which allow self-reference and government itself and be governed by a system of cognitive production from the immaterial labor of the two psychic systems of teaching operation under
}

two systems: economic: human capital and cognitive capital and the Reformed higher education.

Keywords: governmentality, system, higher education, psychic perception system, university teaching.

\section{Introducción}

La noción de "gubernamentalidad" permite al decir Noguera "repensar el lugar de las prácticas pedagógicas" (2009) sobre todo porque permite entrever un uso metodológico para investigar al sujeto educador universitario desde el cómo fue establecido indispensable a partir de su propia acción canalizada por el cálculo de utilidad (interés propio) y la maximización de las ganancias en los nuevos mercados (Hinkelammert, 2005) de la investigación y la docencia, en otras palabras, comprenderlo desde sus maneras de hacer tan importantes porque ellas son útiles en términos económicos al ser rentables y porque a partir de estos cálculos de rentabilidad docente, el mismo educador universitario adquiere no sólo prestigio académico, sino valor de uso de sus discursos representados en un incremento de sus ganancias económicas y sociales, lo que reclama el estudio de las estrategias de gobierno o prácticas que direccionan la conducta de la propia gobernanza de la educación superior y de la subjetividad de quien educa en dos acepciones que propone el autor en mención: la de "pedagogo" y la de 
"maestro", en primer lugar, y la de investigador, en segundo término, como aporte de quien escribe este texto.

El caso que se desea reflexionar, el sujeto universitario colombiano, en esa doble acepción, traída por Noguera a partir de Clemente (1960) exige el reconocimiento desde sus prácticas de gobernamiento. Esos distintos modos de gobierno (Noguera) reclaman un primer momento de estudio, sobre las maneras instituidas que han hecho el conocimiento del propio pedagogo o del maestro de la educación superior en Colombia, a partir de la Ley 30 de 28 de diciembre de 1992, o que ha aflorado en otras designaciones que no obstante su carácter pastoral o de formador moral o de su orientación a la "enseñanza del dogma" se estén hibridizando hasta configurar otro modo de subjetividad, producto de sus propias prácticas de "cuidado de sí", especialmente porque aquel actúa, hoy, no sólo como diseñador y cultivador cultural o como depositario académico que sana "las pasiones del alma" a través del dogma científico, sino que ambas cualidades, la de ser sujeto moral y sujeto del logos se mezclan, atravesando un proceso de transición de un sujeto educador universitario tradicional hacia otro sujeto educador universitario que le lleva a la modernidad o lo saca de la misma (Canclini, 1997) en la trama del intercambio simbólico entre ser trabajador y consumidor (Fumagalli, 2010, Becker, 1983, Vercellone, 2004, Blondeau, et. al., 2004) de sus propios saberes y poderes.

En otras palabras, el educador universitario está inmerso en la modernidad en tanto está sujeto al vaivén de los cambios continuos que le hacen posible. Unos movimientos de entrada y salida de lo que fue y de lo que es, entre lo académico del ayer y lo trabajador y consumidor que es hoy, es lo que permite aseverarlo como sujetado a la modernidad, pues la modernidad como la enfatiza Berman (1988) es una unidad paradójica, porque arroja a la desintegración y a la renovación, a la ambigüedad y a la angustia, que en el docente de la educación superior lo produce como híbrido, o sujetado a condicionamientos que le exigen dejar de pensar y actuar para la productividad académica de sus intereses sociales y científicos e iniciar a enseñar//investigar para la productividad inmaterial de los intereses de los nuevos capitales neoliberales, la del capital humano y la del capital cognitivo.

Es moderno porque está siempre entre dos caminos, ¿Cuándo cesara este trance? ¿En qué se configurará?, son preguntas que por el instante sólo se responden por sí mismas: la modernidad no es de hace dos días, su movimiento "entre caminos" lleva ya quinientos años porque ella misma es la expresión de ese viaje del cambio científico y tecnológico constantes que cada vez se incrementan, ahora con mayor asiduidad por una forma nueva de sociedad, la del conocimiento, que se haya ávida de saberes y del consumo de los mismos. En estos sentidos, se descubre un docente universitario que enseñando e investigando hace obsoleto lo que forma y descubre e inmediatamente enseña e investiga, una vez se consume y hace consumir los productos de su propia obsolescencia, convirtiéndose en un sistema psíquico de percepción, o lo que Luhmann explica como el acabamiento del sujeto y el surgimiento de unidades de información que nutren al sistema, para el caso, al sistema de la educación superior, manteniéndolo moderno.

\section{Sobre las prácticas de gobernamiento de la educación superior en Colombia}

La gubernamentalidad a partir de lo que Noguera comprende como el "conjunto de prácticas y saberes (...) que describen la forma como se constituyeron ciertas racionalidades de gobierno en la modernidad" $(2009$, p. 25) en la educación superior colombiana se ha venido configurando bajo el enunciado "nueva gobernanza" o modos jerárquicos o estatal-estatista o vertical de mando/control dadas antes de la década de los 90 del siglo XX; en ellas prevalecían procesos de gobernar en donde el go- 
bierno central definía sus objetos, acciones e instrumentos preferidos para alcanzar los objetivos que suponían la solución de problemas en cuyas condiciones se ponía más atención al gobernante y a su acción política (Aguilar, 2011).

Esta práctica de gobernanza trae de suyo la noción de gobernamentalidad que permite hacer el análisis de los problemas de gobierno de las décadas de los 50s y hasta los 80 s que por un lado aseguraba la "gobernabilidad de modo autoritario, por lo menos por un tiempo, pero de modo excluyente e inestable" (Lahera, 2004, p.11) y a la vez, el retorno a dos tipos de gobierno de la economía política cuya enunciación se ha distribuido socialmente bajo la connotación de enfoques de desarrollo: el enfoque de la modernización (1945-1965) y el de la dependencia (1965 - 1980) de los estados latinoamericanos propios de la salida de la bipolaridad internacional como se propuso en el Seminario de educación y modernización cultural en Colombia. (2012):

Las prácticas de gobernanza, entonces, se reconocen como modos de gobernar a partir de dos dispositivos de poder: la descentralización y desconcentración que disponen un juego de relaciones de fuerza reconocido en el ejercicio de las políticas públicas como enfoque de gobernanza hacia abajo, o proceso de gobernar desde la política pública y con la gestión pública con escasa participación democrática y más bien impregnada de relaciones de poder fragmentadas con la sociedad, la una instaba al industrialismo (50s) la otra por un intercambio económico desigual (80s) de la periferia al centro (Varcárcel, 2006), ambas trabajaron por el mantenimiento de lo que Aronson (2007) denomina la teoría del capital humano conceptualización que data de las décadas de 1950 y 1960 (...) que vuelve a utilizarse como estrategia teórica para el análisis de los nexos entre la educación y las diversas esferas sociales, especialmente las concernientes a la producción y el trabajo (p. 10).

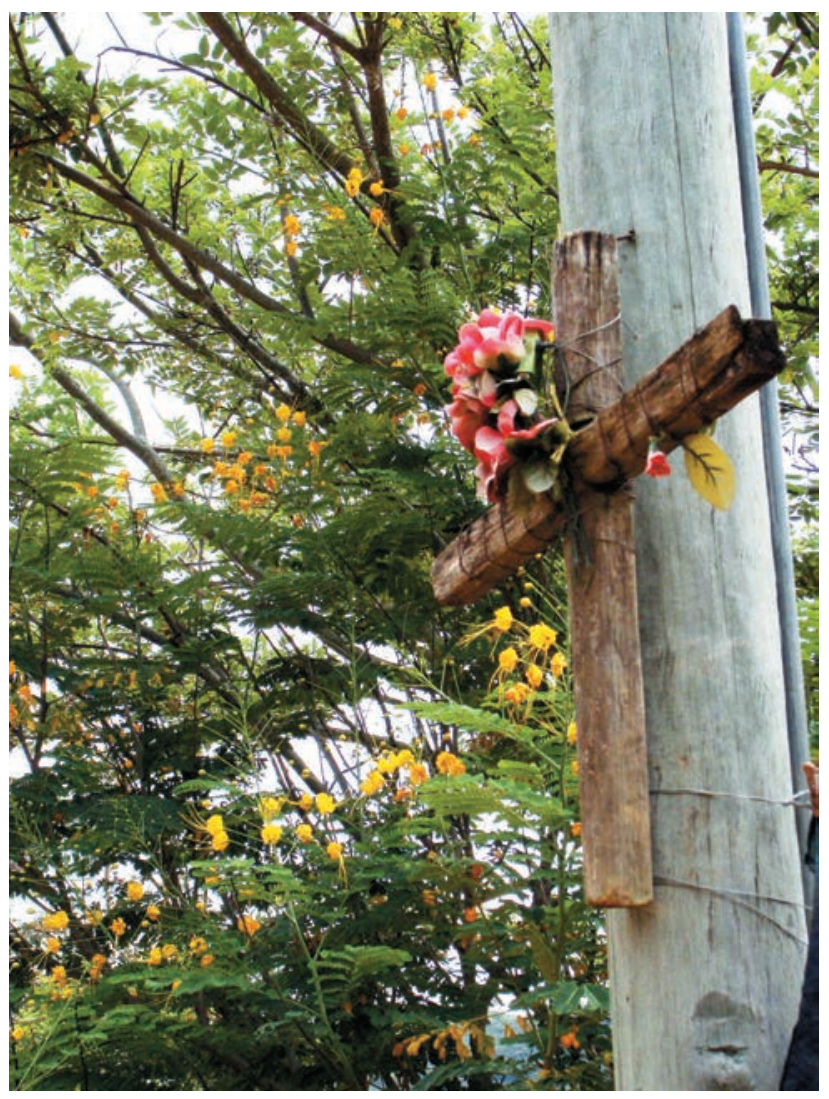

Las formas de gobierno de las décadas de los 90s, entonces inauguran formas de gobierno o prácticas de gobernanza liberal centradas en un enfoque filosófico y político, que Miñana (2002) nombra como posición filosófica de relación entre educación y democracia libertaria o pluralismo segmentado hoy propuesto como "capital humano" (Salcedo, 2013) cuyas condiciones de posibilidad económico/sociales han originado la relación entre derecho a la educación-empresa-economía como coordenadas de lo que al parecer importa, la cooperación iberoamericana para la implementación de una teoría política de la educación, que ha logrado instaurar a un tipo de neoliberalismo en la sociedad del conocimiento, el capital como desarrollo humano, y en él, a la educación como mercancía, impregnadas en principio por las teorías de Theodor Schultz (1983) y Gary Becker (1983; 2003), que ahora se enuncian a partir de cumbres y conferencias iberoamericanas o en otras palabras, en flujos de discurso de poder político cooperativo transnacional. 
Así, la gobernanza en relación con la educación se gesta y se desarrolla en el devenir de una teoría política de la educación que al decir de Alcántara (2012) acontece en "medios académicos anglosajones (...) para designar a la eficacia, calidad y buena orientación en la intervención del Estado (...) haciendo referencia al gobierno interactivo, al gobierno emprendedor, al gobierno socio - facilitador" (pp. 2-3) que no se desarrollaron antes de los 90 s y que ahora son necesarias para permitir relacionales entre lo público-privado-civil a través de la acción pública o de las políticas públicas, lo que Aguilar llama como "los componentes e instrumentos directivos del proceso de gobernar que incluyen el modo o arte de gobernar en un tipo de gobernanza" (2011). Una gobernanza universitaria que devenida de los organismos internacionales fuertemente entrelazados requiere de esa misma dinámica de interconectividad con múltiples agentes sociales que cohesionen de forma rápida las relaciones de poder político, de saber y de ser de las fuerzas administrativas y académicas para producir a partir de la investigación el escenario capaz de permitir la "relación global sectorial (RGS)... herramienta esencial para analizar el cambio y el papel de la acción pública en la regulación del cambio social... y se ajusta el funcionamiento del sector en relación con lo global" (Muller, 2009, 132); esto es, la relación del sector universitario con el estado y la sociedad civil.

Por un lado, el gobierno de la gobernanza de la eficiencia, la eficacia y el rendimiento de cuentas en la universidad constituye el humus que le permite a la acción pública acreditar su capacidad directiva y administrativa en un territorio específico en una comunidad local y en un servicio social concreto, la educación superior, cada vez más globalizado; esto significa, que este tipo de gobernanza universitaria garantiza su posicionamiento local para su aceptación global y su relación con las políticas públicas cuando desarrolla prácticas de decisión/acción capaces de "predecir el sentido del cambio mas no sus modalidades" a través del consenso esto es de gobernanzas convergentes que entrelazan exigencias jurídico/privadas, institucional/sociales capaces de "remover y controlar o menguar, cambiar actitudes, comportamientos, valoraciones y actos; preferencias, relaciones sociales de personas y entre grupos" (Aguilar, 2011), dejando claro para el mundo, la capacidad de involucramiento social que la gobernanza de capital humano cognitivo tiene sobre los problemas de la educación superior, que son, la falta de "a) gestión eficiente del sector público; b) control de los mecanismos y responsabilidad en elecciones, formación de gobierno y práctica gubernamental, y c) establecimiento de la descentralización y transparencia, incluida la participación civil de la sociedad" (Kehm, 2011, p.19).

De esta forma, la disposición de las fuerzas de poder sobre y en la educación superior en Colombia se configuran en una formación discursiva en la que se agrupan acontecimientos de discurso estratégicos internacionales que agencian prácticas de gubernamiento global (Teitelbaum, 2011; Krawczyk, 2002; Toussaint, 2006) y ponen en evidencia aquello que trae Noguera desde Foucault 2007), una gobernanza en donde el Estado es sólo un "efecto móvil de un régimen de gubernamentalidades múltiples" que no sólo devienen de las fuerzas transnacionales, también de la ascesis interior de una universidad proclamada por las fuerzas biopolíticas o mecanismos de poder "atrasada" en tanto, que replegada a sus propias sinergias sin ninguna relación con exigencias y necesidades sociales, políticas y económicas. Tanto, las nuevas formas de gobernamiento dirigidas a la modernización de los Estados nación (descentralización, desconcentración/privatización) (Landinelli, 2009) como los nuevos regímenes normativos sobre la educación superior (eficiencia, eficacia, rendimiento de cuentas/ calidad, certificación, etc.), están configurados 
por esquemas teóricos de economía política (capitalismo humano y capitalismo cognitivo) y matrices de filosofía biopolítica (reguladores sociales como las reformas educativas, por ejemplo) que agencian formas de organización tanto para Estado y en él para las instituciones cuyo poder radica en la conducción, imposición de un régimen, dirigir o dirigirse a alguien, etc. (Noguera, 2009) como la educación superior, y en ella, el sujeto educador que aparece como ya se mencionó, en medio de estas tensiones de fuerza posibles, deseables e indispensables, quizás porque él, en sí mismo, se impone su propio y social reconocimiento y en tal sentido obtiene su constitución subjetiva o manera como hace su propia práctica pedagógica.

De esta estas formas de gobernamiento, la que ha movido con mayor énfasis la transformación de la educación superior desde las décadas de los 90s, promoviendo la descentralización y la condición de privatización posible en el sistema de educación superior, es la reforma (Kraswczyk, 2002; Martínez, 2004; Navarro, s.f.; Braslavsky \& Cosse, 1996; Oliveira, 2009; Ornelas, 2009). La reforma entonces es comprendida como un proceso continuo de «cambios al cambio» como exigencia global a lo local o de sistemas generales a sistemas particulares. Las reformas como modos de gobernamiento sistémico, expanden constantes autorreferentes jurídicos proponiendo políticas de reforma a la educación superior; lo que provoca una constante agenda local de desaceleración de las prácticas de calidad en la enseñanza, en la investigación, en la formación pos gradual acelerando la eficiencia en la gestión institucional. (Narodowski, 2002). La velocidad maquínica (Deleuze, 1985) o productividad de la gestión institucional se impone como condición valorada en el sistema de la educación superior y como forma de gobierno de la producción inmaterial; un gobernamiento sobre la producción cognitiva que en el marco de la reforma debe superar secuentemente su calidad valorada en términos de capital (Palermo, 2002) y en la que el docente universitario resulta rentable en tanto responde a tal velocidad productiva innovando y transfiriendo el conocimiento investigativo para que genere prácticas educativas que distribuyan dicha aceleración, siempre en relación con los sistemas psíquicos de operación investigativa, docencia y extensión.

Las reformas finalmente convierten al sujeto universitario: pedagogo y maestro, en una entidad legal en la que retorna un sujeto juez y al mismo tiempo "reo" u objeto de juzgamiento (Hinkelammert, 2005); en ambos casos, según su sistema de productividad psíquica operativa (Luhmann) u operaciones investigativas y docentes, muestra su existencia siempre rentable en términos neoliberales, cuando éstas son transferibles e innovan el conocimiento empresarialmente exigido para el fomento del desarrollo humano. Por tanto, se instala un sujeto propiedad de sí en relación de uso con su capacidad productiva y la renta que produce desde su "General Intellect" (Marx, 2007, 1999) condensado en los dos sistemas psíquicos de operaciones, calculados, medibles en el sistema de educación superior.

En este sentido, las reformas, no sólo convierten, sino que son convertidas en información de un sistema social que es nutrido desde la educación superior por dos referentes, la investigación y la docencia, como sociedad del conocimiento, que son procesados por el docente universitario, al que se le identifica como entorno y no sujeto. Es entorno en tanto comunica referentes, según la teoría de sistemas, al sistema de educación superior porque en él prevalen datos comunicados por el propio entorno que es nutrido por "irritaciones" que el mismo produce y que no corresponden a necesidades sociales o exigencias humanas; son "irritaciones" autopoiéticas porque produce sus propios elementos fundamentales para ser sistema, así la investigación y la docencia, son autoproducidos por el sistema de educación superior y transmitidos al docente universitario, para su propio mantenimiento y constante 
configuración; la transmisión re-crea los límites y las estructuras internas a la investigación ya la docencia para que el docente navegue, se mueva, piense y actúe.

En otras palabras, la investigación y la docencia universitarias son sistemas psíquicos operativos porque son sistemas autopoiéticos u autoorganización del sistema de educación superior, superando la complejidad del entorno en donde habitan los sujetos que resultan demasiado complejos y a los cuales se les debe proveer de información para que dentro de su complejidad se muevan a partir de un conjunto de relaciones que permitan al sistema superar las irritaciones de la complejidad y mantenerse eternamente re-creador. El sistema se nutre de información de los sistemas psíquicos de operación que lo alimenta de las principales irritaciones del entorno, para que este operacionalice estructuras y límites sobre las representaciones que tiene el entorno de sí, tratando no al entorno ni a los sujetos como sujetos sino como entidades que proveen de información y a las que se les provee información para obturar representaciones. La comunicación nutre el constante cambio del sistema como formación secuente de ideas para mantener la reforma veloz del mismo, afectando los entornos y lo que Luhmann (1991) propone como sistemas psíquicos de operaciones, que desaparecen la inteligencia encarnada del docente universitario, para que él agencie los dos sistemas psíquicos de operaciones: la investigación y la docencia, con arreglo a la información del sistema de educación superior. Así, la investigación y la docencia, son comprendidos como sistemas psíquicos, desde la teoría de sistemas de Luhmann, porque son los eventos del pensamiento del educador universitario que permiten la comunicación con su propio sistema vivo, o cuerpo posibilitando sus propias formas de expresión con el sistema social que es comprendido como el evento de comunicación a través del cual se transmite a sí mismo y a otros, la información derivada del sistema, haciendo autopoiético, es decir, creador de sí mismo, según tenga información comunicada de los sistemas social y vivo, pero siempre como representación de un sistema general, el sistema de educación superior.

Las reformas entronizan en el sistema de educación superior una nueva gobernanza universitaria cuya fuerza radica en la configuración de movilidad, y en ella la cooperación de los docentes no tanto de los estudiantes como los elementos manifiestos de ese nuevo sistema abierto de universidad que requiere el sistema mundo moderno en el marco de convenios bilaterales entre instituciones y países. Unos convenios individuales, limitados y especializados. Característica que evidencia la relación input/ output, del sistema con el entorno y por supuesto la manifestación del carácter limitado de las estructuras individuales, limitadas y especializadas como "normas de transformación" (Luhmann, 1991, p.33) o de variación de las mismas o de la gobernanza clásica que rige aún a la universidad contemporánea.

Ejemplos de este tránsito de gobernanza se halla desde esta posición de movilidad y cooperación en el establecimiento de sistemas de movilidad intra-europea en los años 80s, por ejemplo, a través del programa ERASMUS que modificó la dimensión internacional en las universidades europeas distribuyendo el sistema educativo europeo como sistema autorreferencial mundial:

Los presupuestos, la diversificación de las fórmulas de intercambio, y la extensión del ámbito geográfico... y la puesta en marcha de nuevos programas: TEMPUS con la Europa del Este y más tarde con la cuenca mediterránea; ALFA con América Latina; ATLANTIS con Estados Unidos y más recientemente ERASMUS MUNDUS por la cooperación entre universidades europeas y de cualquier otro país del mundo (Haug, 2012, p.21).

Cobra especial interés, el programa ERAMUS por su capacidad autorreferencial, esto es, la posibilidad de expandirse como eje referenciado y 
referido en diferentes sistemas sin alterarlos utilizando sólo la descripción operativa de la movilidad para que otros desde sus diferencias "no solamente en cuanto a idiomas, sino también a regulaciones, instituciones, titulaciones, calendarios académicos, sistemas de calificaciones, métodos docentes, financiación, etc." (Haug, 2012, p.22) incorporen en sus entornos unas orientaciones y principios de procedimiento informado que quedó limitado en su propia capacidad movilizadora pues era restrictiva a minorías de estudiantes y dependiente de los convenios y de las disciplinas.

Sin embargo, la comunicación del sistema educativo universitario se nutrió (se auto describió, auto observó, auto simplificó) con este tipo de programa, autogenerándose y auto organizando durante 15 años el proceso Bolonia (1999) que sintetiza su capacidad auto poiética en la exigencia de acentuar la movilidad y al mismo tiempo de cualificar la institucionalidad de la educación superior europea. Al respecto Haug (2012) afirma:

Esto implica reformas dentro de las propias universidades, sobre todo una profunda revisión curricular y metodológica de los programas formativos. Al lado de aspectos cooperativos aparecen, también en esta etapa, dimensiones competitivas, con la ambición de preparar mejor a los estudiantes nacionales para el mercado laboral europeo e internacional y fomentar el atractivo de los sistemas y las universidades de cada país en el ámbito europeo, así como mundial (p. 22).

El devenir auto poiético de la educación superior en Europa desde ERAMUS y los programas que se derivan de este hasta la emergencia del proceso de Bolonia (Fernandez \& Serrano, 2009) y la implantación de la modernización de la educación superior como "agenda" a partir de la "Estrategia de Lisboa" que ensambla la producción de conocimiento rentable mediante el establecimiento de una nueva gobernanza universitaria al fomento de la financiación de la

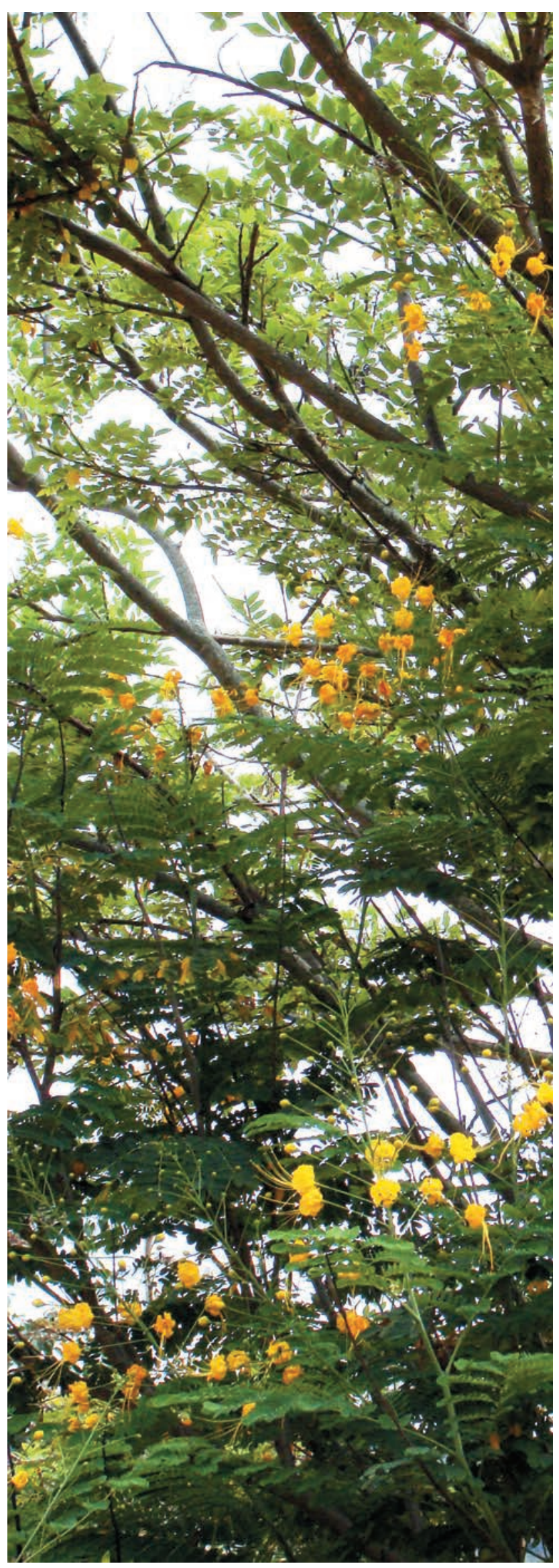


educación superior pública y a la diversificación de la oferta educativa y al acento de ser para toda la vida, es lo que permite su expansión y la enunciación de esta internacionalización del sistema de educación superior europeo como un referente relacional de jerarquías epistémicas sistema-mundo europeo/euro-norteamericano capitalista/patriarcal moderno/colonial sistemamundo capitalista que autorreferencia los matices de la diversidad de entornos situándolos en la relacionalidad de relaciones competitivas que impactan los límites de la misma internacionalización describiéndose ahora como globalización o serie de relaciones de una nueva diferencia directriz ya no de cooperación, sino de competitividad cognitiva que reproduce la manipulación de las diferencias de los entornos en unas relaciones de: "rankings internacionales, (basadas en publicidad, ferias de reclutamiento y cursos específicos para extranjeros) y el desarrollo de la educación superior «transnacional» o «sin fronteras» (exportación de educación superior mediante internet, franchising o campus en el extranjero)" (Haug, 2010, p.22).

En palabras de Martínez (2012), "ese quiebre o ruptura con el rumbo que acontecía... que afecta la estructura y el funcionamiento" (p.21) de la educación es lo que se denomina modernización y que correspondería a un sistema de relaciones de jerarquías epistémicas sistemamundo europeo/euro-norteamericano capitalista/patriarcal moderno/colonial sistema-mundo capitalista que unifica la vida social, para el caso, la producción monetaria de la educación superior a partir de las nuevas actividades de producción/acumulación: investigación, docencia y extensión, que rompen con las antiguas actividades de intercambio/realización académica, a una pluralidad y singularización incontrolable de actividades productivas ligadas a la vida intelectual que busca "transformar la energía libre en energía domada" (FumagaIli, 2010, p.27) y ligar a una serie de relaciones económicas, o en otras palabras, valorar las capacidades cognitivas y las relaciones que éstas generen para la configuración de formas capitalistas de producción.

El sistema educativo ha generado relaciones de comunicación sugestivas (ERASMUS, ATLANTIS, TEMPUS, BOLONIA) de "una política de interpretación de necesidades, mediatizada por discursos expertos (...) señalando la incidencia del retraso en materia de educación en el desarrollo económico de los países" (Martínez, 2004, p.107), que no ha permitido que de la generación de conocimiento se creen las condiciones de posibilidad para la acumulación bioeconómica del capital racional que produce la educación superior.

En estos sentidos, la modernización de la educación superior devenida de referentes relacionales entre jerarquías epistémicas sistema-mundo europeo/euro-norteamericano capitalista/patriarcal moderno/colonial sistemamundo capitalista se ha situado en el sistema mundo educado como la "hybris" (Castro, 2007 o la pretensión de "hacerse un punto de vista sobre todos los demás puntos de vista, pero sin que de ese punto de vista pueda tenerse un punto de vista" (p.83) (todo un sistema), concretamente desde la promulgación de las Declaraciones de la Sorbona y Bolonia (1998 y 1999 respectivamente) que obturan ritmos y tiempos complejos a las relaciones entre el sistema económico cognitivo, y el sistema escolar universitario creando condiciones de posibilidad relacional que afectan las formas y los espacios del entorno de la educación superior europea.

El sistema económico cognitivo o condicionamiento de relaciones (Luhmann) de modernización de la educación superior introduce en la contingencia de las relaciones, selecciones de diferencia económica entre los elementos que devienen del sistema nutrido por la Organización Mundial del Comercio (OMC), el Acuerdo General de Comercio de Servicios (GATS) o la UNESCO, CEPAL, OCDE, BM y la relación que se genera para que se entronice en el entorno de la educación superior como ritmos y tiempos 
que expanden las contingencias necesarias para el orden seleccionado del entorno. Así, la modernización de la educación superior genera una serie de expectativas, deseos por alcanzar que sensibilizan determinadas irritaciones al entorno o yuxtaponen tensiones/relaciones en lo que Barnett (2008) re-conoce como tres tipos de espacio dentro de la universidad:

Espacio pedagógico y curricular... ¿de qué espacios disponen los tutores y los equipos de las asignaturas para probar nuevas pedagogías, con relaciones pedagógicas alternativas?... Espacio de saber o espacio de que disponen los profesionales académicos para satisfacer sus intereses investigadores... y el Espacio intelectual y discursivo o aportes al discurso social y al gran público (p. 21).

Esos condicionamientos o condiciones de posibilidad selectivos del sistema comunicados a los tiempos y espacios del entorno universitario transfieren las yuxtaposiciones, tensiones de relación entre las prácticas administrativas, investigativas, de docencia y extensión; entre los espacios pedagógico/curricular de saber e intelectual/discursivo; por ejemplo el cálculo temporal del aprendizaje introduce las dispositivos de eficiencia y de eficacia a la productividad de la gobernanza universitaria evidente en el entorno de la educación superior en el modelo ECTS (European Credit Transfer System) que a su vez, traslada al centro del proceso educativo el aprendizaje dejando en una posición de aparente rezago productivo a la enseñanza y des-territorializa el aula a espacios de práctica directa con las situaciones sociales modificando los roles y las relaciones de los docentes y de los dicentes en donde "el alumno se verá obligado a asumir un papel mucho más activo, autónomo y menos dirigido, mientras sus profesores pasarán a asumir un papel de facilitador" (Fernández, 2006 p.390) que implican modificaciones a las metodologías (Gros \& Romaña, 2004) centradas más en el trabajo individual del estudiante, acentuando la des-temporalización de la enseñanza por una disminución del tiempo aula fracturando los procedimientos de evaluación que deben girar ya no en "evaluar únicamente conocimientos sino más bien de ser además capaces de valorar en qué medida se ha conseguido un cambio de actitudes y se ha logrado un desarrollo de procesos de calidad" (Fernández, 2006, p.391), e incorporando procesos de evaluación permanente a la propia institución de educación superior a través de visitas de pares o de dispositivos de control sobre la productividad/titulación según los perfiles profesionales.

El sistema de la educación superior comunica una serie de relaciones de eficiencia y eficacia sobre la enseñanza, el aprendizaje, las metodologías, la evaluación; la administración de los recursos, las fuentes de financiación instalando prácticas de orden productivo capitalista que agencian las diferencias sobre el diseño y el control de los entornos, convirtiéndolas en relaciones de autoproducción de un sistema económico cognitivo o condicionamiento de relaciones de modernización de la educación superior.

Diríase que el sistema económico cognitivo o condicionamiento de relaciones de modernización de la educación superior re-produce en Europa, un entorno que adquiere el sentido de Espacio Europeo de Educación Superior, EEES, que evidencia la capacidad interpretativa del sistema sobre el entorno. El sistema económico cognitivo distribuye la condición de un espacio de educación superior como límite del entorno, esto es como "la realidad de un más allá y la posibilidad de rebasarla" (Luhmann, 1996, p.51). En otras palabras, se expande la idea (sistema) de un ente unificador de entorno de educación superior a través de la categoría tiempo que penetra la categoría espacio de educación superior mediante los ritmos del mundo académico (disciplinas, cátedras, departamentos, facultades, planes de estudios, proyectos de investigación, entre otros. 


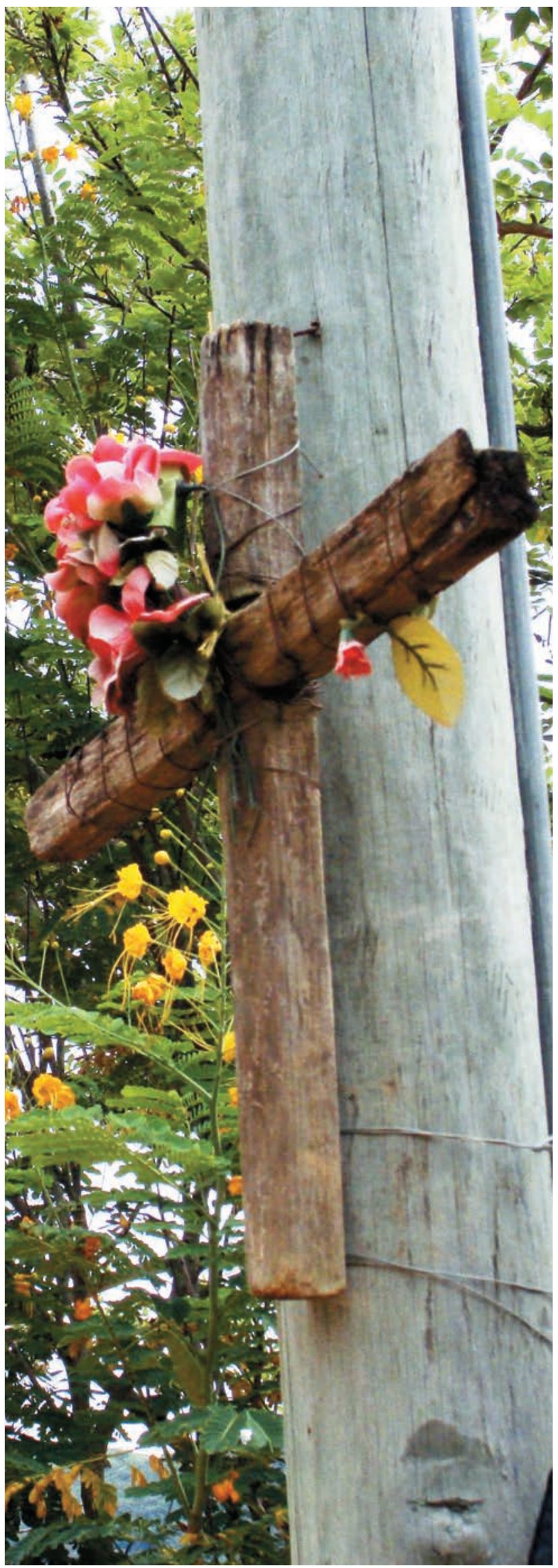

La categoría espacio/temporal del ritmo, se pone como fuente metafórica que permite describir cómo crea el sistema las condiciones de posibilidad (condicionamientos) para superar el límite que distribuye el sistema educativo y mediar la modernización de la educación superior; : Así, el ritmo es comprendido como dispositivo que relaciona "un «detrás» y «un más allá», esto es lo que vehicula en el interior del límite, el cambio del entorno, en tanto adapta cualquier movimiento (espacialidad) del entorno a un conjunto complejo de actividades coordinadas (temporalidad): "formación continua, flexible, transversal y psicoafectiva de un profesional todo terreno, capaz de estar en todo momento a la altura y al tanto de las necesidades ingobernables de un mercado laboral cada vez más imprevisible" (Fernández, 2009, p.77), conforme a las necesidades del sistema económico cognitivo que posibilita situar a la Universidad al servicio de las empresas, subordinando su financiación a la previa obtención de "fuentes de financiación externa".

El ritmo, dispositivo conector del límite como condición de superación instala un régimen de veridicción, la flexibilidad, en el entorno. Esto otorga sentido social aparente a la modernización de la educación superior. La Red Eurydice, conformada por 37 unidades nacionales con base en los 33 países participantes en el programa de aprendizaje a lo largo de la vida de la UE (Estados Miembro de la UE, países de la AEL, Croacia y Turquía) y coordinada por la Agencia Ejecutiva en el ámbito Educativo, Audiovisual y Cultural de la UE, con sede en Bruselas ${ }^{1}$, ofrece la siguiente información que permite reconocer la aparente dimensión social del ritmo a través de la flexibilidad. Un dimensión que esta materializada (relación sistema/entorno) por la percepción moderna de "participación" y "dimensión social", esto es, como categorías que producen ritmos de "cooperación", "retos", "progreso", "fe-

1 Bruselas es "la principal sede administrativa de la Unión Europea (UE). Es también sede de la Comisión Europea, del Consejo Europeo, del Consejo de la Unión Europea y una de las dos sedes del Parlamento Europeo. La ciudad también es la sede política de la Organización del Tratado del Atlántico Norte (OTAN)" (Bruselas, Wikipedia, tomado el 17 de septiembre de 2012 en http://es.wikipedia. org/wiki/Bruselas). 
nómeno social" por todos esperado como necesidades de modernización, a través de la actual fase económica que hace de la educación el principal referente autopoiético de la modernización de la educación superior.

La flexibilidad se distribuye como una serie de acciones coordinadas (ritmo) que operacionaliza la percepción de igualdad de condiciones para cursar estudios de educación superior y su correspondiente aceptabilidad por los consumidores del entorno, los aprendizajes. Así la UE incrementó la participación de la población a los estudios superiores creando políticas ministeriales que hicieron hincapié en que el "alumnado debería poder finalizar sus estudios sin ningún tipo de obstáculo relacionado con su origen social o su situación económica" (Comunicado de Londres 2007, p. 5), asunto que incorporan los países miembros de Eurydice creando condiciones de posibilidad (condicionamientos) alternas; la red en mención descubre que dichas prácticas alternas para incrementar la participación en la educación superior se ha convertido en un patrón dominante de la Europa occidental; el mapa que sigue reconoce esta geografía de condicionamientos.

\section{El sujeto universitario: entre el pedagogo y el maestro y el investigador. Su hibridación}

Dichas prácticas de gobernamiento, devienen en el ejercicio de prácticas de enseñanza superior cuya urdimbre enunciativa se prescribe en una formación integral (Ley 30. Art. 6) para el "espíritu reflexivo y la autonomía personal" (Ley 30. Art. 4,), que se convierten en una especie de "lettres de cachet" o tecnologías de poder o leyes que exigidas por las instituciones disciplinarias (presidencia, senado, cámara de representantes y ministerio de educación nacional) obligan a la realización de una acción determinada y que se instituyen mediante "estatutos del profesor universitario" o conjunto de regulaciones sobre los sentidos de pertenencia laborales dispuestas por una serie de enunciados considerados signos fuerza instituidos ca- paces de normalizar al poder individualizante del educador universitario, en las facetas del saber al orden del Estado o en otras palabras en esa realidad específica y discontinua correlativa a una manera de gobernar.

Esta perspectiva Foucultiana permite entrever que la realización del sujeto de la educación superior está atravesada en su configuración por problemas de gobernamiento con sentido político y sentido económico que en primer lugar sólo se han comprendido en el sentido estatizado fijado en pre-ocupaciones de regulación social:

Graves problemas en la educación superior -en particular en Colombia-, relacionados con una cobertura insuficiente, una calidad muy desigual de las instituciones y de los programas, y una exigua racionalidad de la oferta que, en esas condiciones, se orienta más por la rentabilidad de las formaciones ofrecidas que por las necesidades sociales. (Misas, 2004, p.13).

Una pequeña muestra de que en Colombia la educación superior es estratégica, concretamente por la apertura de relaciones económicas, políticas, sociales y culturales que la disponen en una nueva historia de sus prácticas centrada en una mayor cercanía con las realidades que investiga y enseña esta vez en un nuevo plano: relaciones de poder y de saber conectadas con la ciencia, la tecnología y la sociedad productiva y consumidora. La educación superior a través del sector académico colombiano evidencia su presencia estratégica en relación con los sectores productivos y políticos o la educación superior para el desarrollo en la que se configuran nuevos sujetos de la educación superior cuyas realizaciones pedagógicas y de maestría se mezclan conectadas por las relaciones de producción cognitiva, que hoy, son la nueva metáfora de la fuerza de trabajo.

La Ley 30 de 1992 constituye un dispositivo de gobernamiento de la educación superior. En 
ella, se proclaman las prácticas de un gobierno posterior a la educación media o secundaria diferentes en varios niveles de pastoreo universitario, a saber:

Artículo $9^{\circ}$ Los programas de pregrado preparan para el desempeño de ocupaciones, para el ejercicio de una profesión o disciplina determinada, de naturaleza tecnológica o científica o en el área de las humanidades, las artes y la filosofía. También son programas de pregrado aquellos de naturaleza multidisciplinaria conocidos también como estudios de artes liberales, entendiéndose como los estudios generales en ciencias, artes o humanidades, con énfasis en algunas de las disciplinas que hacen parte de dichos campos.

Artículo 10. Son programas de postgrado las especializaciones, las maestrías los doctorados y los postdoctorados.

Artículo 11. Los programas de especialización son aquellos que se desarrollan con posterioridad a un programa de pregrado y posibilitan el perfeccionamiento en la misma ocupación, profesión, disciplina o áreas afines o complementarias.

Artículo 12. Los programas de maestría, doctorado y post-doctorado tienen a la investigación como fundamento y ámbito necesarios de su actividad. Las maestrías buscan ampliar y desarrollar los conocimientos para la solución de problemas disciplinarios, interdisciplinarios o profesionales y dotar a la persona de los instrumentos básicos que la habilitan como investigador en un área específica de las ciencias o de las tecnologías o que le permitan profundizar teórica y conceptualmente en un campo de la filosofía, de las humanidades y de las a artes. (Ley 30).

La normativa traza una jerarquía de gobernamiento sobre la formación que debe suministrar el sistema de educación superior y a su vez, expone las exigencias de un país moderno, en tanto, configure condiciones de posibilidad educativas en el sector terciario de la educación. Plantea una serie de información concentrada en una dinámica arbórea que clasifica y masifica la arborización de la educación superior configurando un sistema constante de "entradas" y "salidas" de nunca acabar para quien desee no sólo educarse sino educar e investigar.

En los cuales, la presencia del sujeto de la enseñanza superior se reclama en esa misma gubernamentalidad normalizada enunciada en la Ley 30, capítulo III "Del personal docente y administrativo". En esta disposición normativa, se hallan rasgos de una subjetividad docente que quizás entraña un sujeto pedagogo y un sujeto maestro, pero en todo caso, más un docente como capital humano, en tanto educador de sí mismo, como productor de calidad de enseñanza y educador de otros o cualificador de las capacidades humanas para la producción. (Vaillat, 2005) siempre en relación y tensión con sus funciones: la del ejercicio investigativo y del quehacer de la docencia. (Barnett, 2008).

Los rasgos del sujeto docente, desde el poder instituido se establecen condicionados por una visión jurídica o en palabras de Foucault por unas tecnologías de vigilancia centradas en definir concreciones de individuación docente fijas en relaciones de eficacia en las que se presuponen sus actividades, las cuales quedan replegadas a un imaginario que supone una identificación del docente a través de:

a. Régimen de vinculación, promoción, categorías, retiro y demás situaciones administrativas.

b. Derechos, obligaciones, inhabilidades, incompatibilidades, distinciones y estímulos.

c. Establecimiento de un sistema de evaluación del desempeño del profesor universitario.

d. Régimen disciplinario. 
Artículo 76. El escalafón del profesor universitario comprenderá las siguientes categorías:

- Profesor Auxiliar.

- Profesor Asistente.

- Profesor Asociado.

- Profesor Titular. (Ley 30. Art. 75).

En otras palabras, se habla de docente desde unas condiciones de vigilancia que suponen su defensa como trabajador "cognitivo" según regímenes de personal docente y administrativo como una estratificación que se construye y designa desde una cultura de ámbitos administrativos que normalizan unas formas de subjetividad con arreglo a fines racional-económicos (Martínez, 2005) que limitan y al mismo tiempo liberan las percepciones de promoción del saber, haciendo identificable el cuidado de sí a partir del control de su patrimonio cognitivo, en otras palabras, se crean las condiciones de posibilidad para que el sujeto "cumplidor" del estatuto se transforme en docente mediante lo que Foucault concibe como una anatomía política o mecánica capaz de volver al cuerpo del sujeto de la enseñanza superior en docente o sujeto apto, útil, eficiente, dócil y obediente a través del cual se integran sistemas educativos; económicos. El sujeto de la educación superior no sólo es práctico pedagógico o conductor de actitudes, u orientador de conductas, ni un teórico cognitivo que transpira ciencia en los actos de enseñanza superior, sino capaz de gobernarse a sí mismo desde la acción pedagógica y la enunciación teórico-investigativa se forja a sí mismo, es docente porque en él se ejercita la docencia entendida como la forma de conducción de su propia conducta en tanto de él y desde él, se desprende su propia condición.

\section{Conclusiones}

El sistema de educación superior reconoce una "trama de poder-gubernamentalidad-go- bierno de sí y gobierno de los otros-relación de sí consigo" (Foucault, 2002, p. 274). El docente universitario es uno de los sujetos de la educación superior, en él se articulan relaciones de poder y de saber cognitivo, político y económico capaz de dirigir la espiritualidad de las almas contemporáneas deseosas de educación, de productividad cognitiva y de consumo de libertad que provee la educación superior como transfiguración de la seguridad gubernamental.

La trama de poder-gubernamentalidad se expande a partir de los sistemas de educación por una serie de dispositivos de calidad docente o "todas aquellas actuaciones destinadas a mejorar los conocimientos, competencias y habilidades del profesor universitario, todas ellas con repercusión inmediata en el aula y donde el papel de la innovación educativa ocupa un lugar imprescindible" (Arranz Val, P. 2007, p. 158), que hacen de la docencia universitaria una práctica cognitiva competitiva, es decir, creadora del condicionamiento para afectar el desarrollo social, cultural y económico de la población que asume una educación para lo superior. La docencia constituye un autorreferente de la expansión de la modernización de la educación superior como dispositivo del proceso de producción capitalista cognitivo (Stubrin \& Gentili, 2009) en donde su expansor ${ }^{2}$ o procesador dinámico de la señal cognitiva, el educador universitario, es visible en tanto disponga sus autorreferentes vitales y profesionales a tal fin. Así, para Colombia, "la formación profesional es garantía de bienestar, progreso, desarrollo y solidaridad" (ASCUN, 2010, p.1) y sinónimo de un docente eficiente y eficaz, capacitado para rendir cuentas cognitivas ante el futuro de su nación y del globo.

Unido a la docencia, este poder-gubernamentalidad, se reproduce en otro tipo de pro-

2 Se usa el concepto "expansor" metafóricamente para expresar el aumento del rango de la señal autorreferente del docente universitario en la sociedad del conocimiento. Es una presencia aumentada por el sistema de educación superior, expandida en la intimidad de la sociedad. 
ductividad docente, la investigación. Ésta se ha convertido en un dispositivo de control sobre el pensamiento y la acción de producción de conocimiento en donde el agenciamiento de intereses singulares sobre fenómenos de la realidad empiezan a difuminarse del educador universitario a los sistemas de investigación que informan lo que debe investigarse bajo condicionamientos temáticos, regionales y financieros, en otras palabras, económico/políticos. Unos condicionamientos que siendo externos a la universidad, ella los incorpora a sus sistemas de condicionamientos normativos, vía reglamentos profesorales, cediendo su autonomía, o mejor permitiéndose "sentir" que su autonomía se expande y en este sentido, haciendo percibir al docente investigador la condición de investigar las realidades operativas que el sistema indica para permanecer convocados a participar en la construcción social.

Cientos de convocatorias configuran con mayor fuerza sistémica, la producción de conocimiento del docente universitario. El uso y desuso de convocatorias traduce un proceso constante de conexión y desconexión del pensamiento y acción docente que acelera el ritmo de productividad a temporalidades que estas determinan, que para el sistema síquico del docente, se traduce en una contracción de sus oportunidades e intereses investigativos y una expansión de los condicionamientos económico/políticos nacionales e internacionales. Los sentidos de producción de conocimiento, las búsquedas de fenómenos y sus maneras de comprenderlos inician giros epistemológicos y metodológicos concentrados en la satisfacción de tiempos más cortos para investigar y en los recursos financieros; quizás las realidades y sus problemáticas resulten trazadas en planes de investigación del sistema de investigación nacional e internacional sin ser transformadas, el interés se halla en describirlas como una forma envolvente de comprenderlas para darles más un tratamiento económico/político que satisfaga la especulación de derechos, la exaltación de políticas, pauperizando en la sociedad, la presencia del investigador universitario.

Los sistemas psíquicos del docente, la docencia y la investigación, resultan gubernamentalizados. Ellos son la vía de gobierno del pensamiento y la acción docente e investigativa. Esto no se traduce en una perspectiva psicológica en la que el comportamiento y la mente del individuo resulten a conductados, sino en el sentido del pensamiento y acción del educador universitario que excede lo puramente psicológico. Es en este sentido, el sistema psíquico de percepción docente, un dispositivo de poder y de saber gubernamental o conjunto de prácticas reguladas que pueden formar y transformar la enseñanza y la producción de conocimiento desde los condicionamientos económico/políticos vía comunicación de convocatorias públicas y privadas, nacionales e internacionales, de normas, conectividad online y reglamentos docentes universitarios expandidos por la fuerza de los condicionamientos de la sociedad del conocimiento; última expresión en la que no se reconoce sino un todo social siempre interesado en conocer vía educación superior.

\section{Referencias}

Aguilar, L (2011) Gobernanza local y educación: la supervisión escolar. Recuperado de www.youtube.com

Alcántara, A. (s.n.) Gobernanza, gobierno y gobernabilidad en la educación superior. En Lerner, B. et. Al. (s.n.). Gobernabilidad y Gestión Pública en el México del siglo XXI. México, IIS-FCPS-UNAM. En: Seminario: Educación y modernización cultural en Colombia. Doctorado Interinstitucional en Educación. Universidad Pedagógica Nacional. 2012.

Aronson, P. (2007). El retorno de la teoría del capital humano. Revista Fundamentos en Humanidades, No.8, Número 2 (16), 9-26. Argentina, Universidad Nacional de San Luis. En: Seminario: Educación y modernización cultural en Colombia. Doctorado Interinstitucional en Educación. Universidad Pedagógica Nacional. 2012.

Arranz, Val, P. (2007): Los sistemas de garantía de calidad en la Educación Superior en España. Propuesta de un modelo de acreditación para las titulaciones de 
Grado en Empresa. Tesis Doctoral, Universidad de Burgos. En: Castilla, F. (2011). Calidad docente en el ámbito universitario: Un estudio comparativo de las universidades andaluzas. Revista de Educación en Contabilidad, Finanzas y Administración de Empresas. No. 2, 157-172.

ASCUN. (2010). Hacia una nueva dinámica social de la Educación Superior. Documento de políticas. 2010 1014. Bogotá: Autor.

Barnett, R. (2008). Para una transformación de la universidad. Nuevas relaciones entre investigación, saber y docencia. Barcelona: Ediciones Octaedro.

Becker, G. (1983). El capital humano. Madrid: Alianza.

(2003). Capital humano en la nueva sociedad. Presentación en la Fundación DMR. Recuperado de http://www.fundaciondmr.org/textos/conferencia01d.

Berman, M. (1988). Todo lo sólido se desvanece en el aire. La experiencia de la modernidad. Madrid: Siglo XXI.

Blondeau, O., Dyer, N., et. al. (2004). Capitalismo cognitivo, propiedad intelectual y creación colectiva. Madrid: Traficantes de sueños.

Braslavsky, C. \& Cosse, G. (1996). Las actuales reformas educativas en América Latina: cuatro actores, tres lógicas y ocho tensiones. Buenos Aires: Flacso.

Canclini, N. (1997). Culturas Híbridas. Estrategias para entrar o salir de la modernidad. Grijalbo: México.

Clemente de Alejandría [200]. El pedagogo. Traducción de Joan Sariol Díaz. Editorial Gredos, Madrid. 1980. En: Noguera, C. (2009). La noción de "gubernamentalidad" y su utilidad para pensar las prácticas pedagógicas. En: Revista Educação e Realidade. 34 (2), 21 - 33.

Consejo Nacional de Política Económica y Social. (2009). Política Nacional de Ciencia, Tecnología e Innovación. Bogotá: Autor.

Deleuze, G., \& Guattari, F. (1985). El Anti-Edipo. Capitalismo y Esquizofrenia. Barcelona: Paidós.

Díaz, M. (2002). Flexibilidad y Educación Superior en CoIombia. Bogotá: ICFES.

Fernández, C. (2006). La educación comparada y sus potencialidades para afrontar el estudio del espacio europeo de la educación superior. En: Revista Española de Educación Comparada, 12, 389 -405. Facultad de Ciencias de la Educación. Universidad de Oviedo.
Fernández, L. (2009). El Plan Bolonia. Madrid: La Catarata.

Fernandez, C., y Serrano, C. (2009). El Plan Bolonia. Madrid: La Catarata.

Fumagalli, A. (2010). Bioeconomía y capitalismo cognitivo. Hacia un nuevo paradigma de acumulación. Roma: $\mathrm{Ca}$ rocci Editore.

Gros, B., \& Romañá, T. (2004). Ser profesor. Palabras sobre la docencia universitaria. Barcelona: Octaedro.

Haug, G. (2012). La internacionalización de la educación superior: más allá de la movilidad europea. La Cuestión universitaria, 6, 20-29. Recuperado en: http://www. lacuestionuniversitaria.upm.es/web/grafica/articulos/imgs_boletin_6/pdfs/LCU-6-3.pdf

Hinkelammert, F. (2005). El sujeto y la ley. El retorno del sujeto reprimido. Costa Rica: Euna.

Kehm, B. (2011). La gobernanza en la educación superior. Sus significados y su relevancia en una época de cambios. Barcelona, Octaedro. En: Seminario: Educación y modernización cultural en Colombia. Doctorado Interinstitucional en Educación. Universidad Pedagógica Nacional. 2012.

Kraswczyk, N. (2002). La reforma educativa en América Latina desde la perspectiva de los organismos internacionales. Revista Mexicana de Investigación Educativa, 7 (16).

Landinelli, J. (2009). Las finalidades públicas de la universidad en el contexto de la globalización. En: Gentilli, P. Políticas de privatización, espacio público y educación en América Latina. Rosario: Homo Sapiens.

Luhmann, N. (1991). Sistemas sociales. Lineamientos para una teoría general. México: Universidad Iberoamericana.

Marx, C. (1999). El Capital I. Crítica de la Economía política. México: Fondo de Cultura Económica.

. (2007). Manifiesto comunista. Valladolid: Ediciones Maxtor.

Martínez, A. (2004). De la escuela expansiva a la escuela competitiva. Dos modos de modernización en América Latina. Bogotá: Anthropos.

Martínez, (2005). La educación en América Latina: entre la calidad y la equidad. Barcelona: Octaedro.

. (2012). Currículo y modernización. Cuatro décadas de educación en Colombia. Bogotá: Magisterio, 
Ministerio de Educación Nacional (1992). Ley 30 de 1992. Artículos 1-143. Bogotá, Colombia: Autor.

Miñana, C., y Rodríguez, G. (2002). La educación en el contexto neoliberal. s.l. En: Seminario: Educación y modernización cultural en Colombia. Doctorado Interinstitucional en Educación. Universidad Pedagógica Nacional. 2012.

Misas, A. (2004). La educación superior en Colombia: análisis y estrategias para su desarrollo. Universidad Nacional de Colombia. Bogotá. En: Seminario: Educación y modernización cultural en Colombia. Doctorado Interinstitucional en Educación. Universidad Pedagógica Nacional. 2012.

Muller, P. (2009). Las políticas públicas. Bogotá, Universidad Externado de Colombia. En: Seminario: Educación y modernización cultural en Colombia. Doctorado Interinstitucional en Educación. Universidad Pedagógica Nacional. 2012.

Navarro, J. (s.f.). Las reformas educativas como reformas del Estado: América Latina en las dos últimas décadas. En: El estado de las reformas del Estado en América Latina. Mayol.

Narodowski, M. (2002). Nuevas tendencias en políticas educativas. Estado, mercado y escuela. Buenos Aires: Granica.

Noguera, C. (2009). La noción de "gubernamentalidad" y su utilidad para pensar las prácticas pedagógicas. En: Revista Educação e Realidade. 34 (2), mai/ago. Pp. 21 - 33.

Oliveira, D. (2009). Reformas educativas y redefinición de las fronteras entre lo público y lo privado. En: Gentili, P. (2009). Políticas de privatización, espacio público y educación en América Latina. Rosario: Homo Sapiens.

Ornelas, J. (2009). Neoliberalismo y capitalismo académico. En: Gentili, P. (2009). Políticas de privatización, espacio público y educación en América Latina. Rosario: Homo Sapiens.

Palermo, Z. (2002). Políticas de mercado/Políticas académicas. Crisis y desafíos en la periferia. En: Walsh, C., Schwy, F., y Castro, S. (2002). Indisciplinar las Ciencias Sociales. Geopolíticas del conocimiento y colonialidad del poder. Perspectivas desde lo andino. Quito: Abya-Yala Ediciones.
Salcedo, J. (2012). El desafío de la gobernanza en la cotidianidad de la universidad desde las reformas a la educación superior en América Latina. Casos Colombia y Brasil. En: Seminario: Educación y modernización cultural en Colombia. Doctorado Interinstitucional en Educación. Universidad Pedagógica Nacional. 2012.

. (2013). La forma neoliberal del capital humano y sus efectos en el derecho a la educación. Revista Actualidades Pedagógicas. No. 61, 113 - 138. Bogotá: Universidad de La Salle.

Schultz, T. W. (1983). La inversión en capital humano. Revista Educación y Sociedad, 8 (3).

Stubrin, F., \& Gentili, P. (2009). Estrategias de mercantilización de la educación y tiempos desiguales de los tratados de libre comercio: el caso Brasil. En: Gentili, P. (2009). Políticas de privatización, espacio público y educación en América Latina. Rosario: Homo Sapiens.

Teitelbaum, A. (2011). Los golpes de Estado del capital financiero transnacional. Revista Izquierda.

Toussaint, E. (2006). Banco Mundial. El golpe de Estado permanente. Ginebra: El viejo topo.

Vaillat, D. (2005). Reformas educativas y rol de docentes. En: Protagonismo Docente en el cambio educativo. Revista PRELAC, 1.

Varcárcel, M. (2009). Génesis y evolución del concepto y enfoques sobre el desarrollo. Documento de investigación. Lima, Pontificia Universidad Católica del Perú. En: Seminario: Educación y modernización cultural en Colombia. Doctorado Interinstitucional en Educación. Universidad Pedagógica Nacional. 2012.

Vercellone, C. (2004). Las políticas de desarrollo del capitalismo cognitivo. En: Blondeau, O et. al. (2004). Capitalismo cognitivo, propiedad intelectual y creación colectiva. Madrid: Traficantes de sueños. 